\title{
Study of dynamic characteristics of slow-changing process
}

\author{
Yinong $\mathrm{Li}^{\mathrm{a}}$, Ling Zheng ${ }^{\mathrm{b}}$ and Bangchun Wen ${ }^{\mathrm{c}}$ \\ ${ }^{a}$ State Key Lab. of Mechanical Transmission, \\ Chongqing University, Chongqing, 400044, China \\ Tel.: +86023 65106094; Fax: +86023 65106195; \\ E-mail:ynli@cqu.edu.cn \\ ${ }^{\mathrm{b}}$ Department of Automobile Engineering, Chongqing \\ University, Chongqing, 400044, China \\ ${ }^{\mathrm{c}}$ School of Mechanical Engineering, Northeastern \\ University, Shenyang, Liaoning, 110006, China \\ Tel.: +86024 23906311; Fax: +86024 23890448; \\ E-mail: bcwen@mail.neu.edu.cn
}

Received 21 April 1999

Revised 25 January 2000

\begin{abstract}
A vibration system with slow-changing parameters is a typical nonlinear system. Such systems often occur in the working and controlled process of some intelligent structures when vibration and deformation exist synchronously. In this paper, a system with slow-changing stiffness, damping and mass is analyzed in an intelligent structure. The relationship between the amplitude and the frequency of the system is studied, and its dynamic characteristic is also discussed. Finally, a piecewise linear method is developed on the basis of the asymptotic method. The simulation and the experiment show that a suitable slow-changing stiffness can restrain the amplitude of the system when the system passes through the resonant region.
\end{abstract}

\section{Introduction}

Many physical and engineering problems have features that may be qualitatively described by nonlinear vibration. The natural frequencies of these systems can be combined through nonlinear interactions so as to produce internal resonance. Although much work has been done on the subject, the natural frequencies are assumed to be time independent with the resonant conditions satisfied for all time. In fact, many vibration problems in engineering are governed by the systems with slow changing parameters. This system is a typical nonlinear system in which the masses, stiffness, damping and exciting force vary slowly with respect to time compared with an ordinary parameter system. For example, in the process of a rocket launching, the mass of the rocket decreases gradually as the fuel continuously burns. Thus the natural frequency and amplitude vary slowly with time. In the running process of a lifter, not only the length of the rope changes slowly with time but also the mass and the stiffness of the vibration system vary slowly. Another example is provided by cracks of rotors in machines, that increase gradually with time development of the cracks relates to the change of the rotor stiffness, thus the process of the crack development has the feature slowly-changing stiffness [1]. Additionally some intelligent structures are parameters such as mass, stiffness, damping and exciting force that change slowly with respect to time due to vibration and large deformation. "Slow-changing" means that the parameters change slowly with respect to the time of a single vibration period, and the obvious change can be seen only in following more periods.

In some intelligent structures, we also need to control their vibration, noise, deformation, or shape. In these processes, the time delay and unstable working condition must be considered in order to avoid the resonance of the structures, to decrease the amplitude of the vibration and to improve the speed of passing through the resonant region. At the same time, the nonlinear dynamic response requires attention to stability.

The nonlinear vibration problems can often be solved by the perturbation theory, but the ordinary perturbation theory cannot handle this problem because of mathematical difficulties. Y.A. Mitropoliski [7] advanced an asymptotic method of system with slow-changing parameters. Kevorkian [3] considered the model problem of two oscillators with weak nonlinear coupling and either constant or slowly varying frequencies to survey perturbation solution techniques based on the idea of multiple-variable expansion applied to problems that exhibit resonance. He also summarized the perturbation techniques and proposed the first order equations that described various weakly non-linear vibration with slow changing parameters [4]. For this class of sys- 


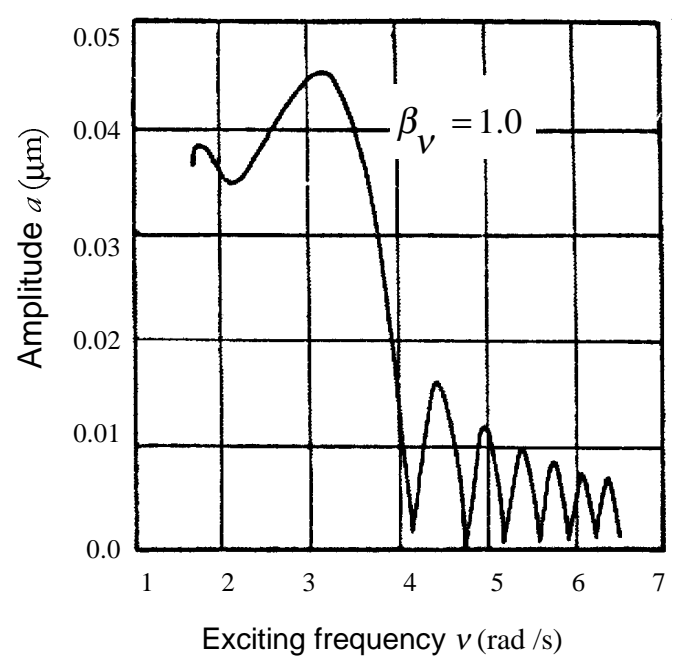

(a)

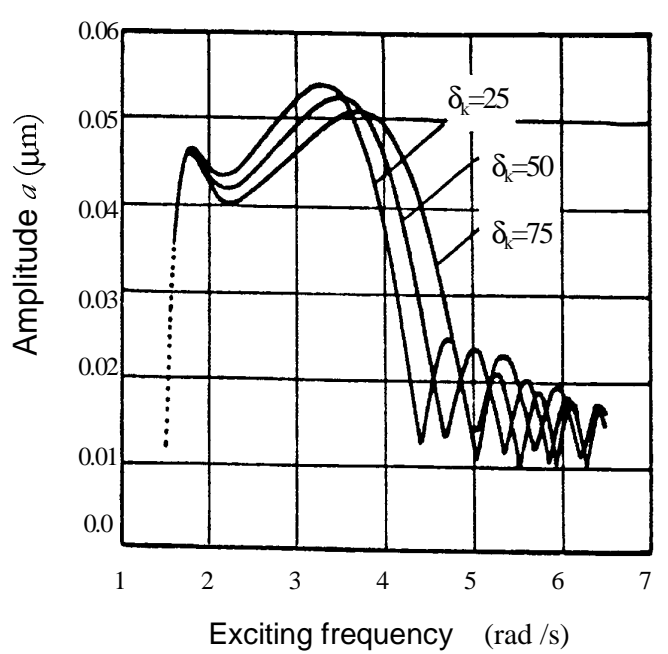

(b)

Fig. 1. (a). The response of the system with slow-changing exciting frequency. (b). The response of the system with slow-changing stiffness.

tems, an explicit approximate solution was derived, based on the technique of freezing slowly changing parameters [6]. In addition, the generalization of the EKB method is used to solve Duffing oscillators with slowly varying parameters [5]. Recently, Bosely [2] used canonical averaging techniques to deal with these systems in Hamiltonian standard forms to very high order and studied their adiabatic invariance.

In this paper, the slow-changing process in the intelligent structures is analyzed and the piecewise linear method is developed on the basis of the asymptotic method.

\section{Dynamic characteristic}

A system with slowly changing parameters is given by a multi-degree-of-freedom system of the form:

$$
\begin{aligned}
& {[M]\{\ddot{x}\}+[C(\tau)]\{\dot{x}\}+[K(\tau)]\{x\} } \\
= & \{f(\tau, \theta, x, \dot{x})\}
\end{aligned}
$$

where $[M],[C]$ and $[K]$ are the mass matrix, damping matrix and stiffness matrix of the slow-changing structure, respectively; $\{x\}$ is a displacement vector and the over dots refer to time dervafca.

In order to improve the calculate accuracy, the slowchanging process can be divided into some sections, i.e. the process of piecewise linear variation, thus the slowchanging mass $m_{i j}(\tau)$, damping $c_{i j}(\tau)$ and stiffness $k_{i j}(\tau)$ can be written as follows:

$$
\begin{gathered}
m_{i j}(\tau)= \begin{cases}m_{1}(\tau), & 0<\tau \leqslant \tau_{1} \\
m_{2}(\tau), & \tau_{1}<\tau \leqslant \tau_{2} \\
& \cdots\end{cases} \\
c_{i j}(\tau)= \begin{cases}c_{1}(\tau), & 0<\tau \leqslant \tau_{1} \\
c_{2}(\tau), & \tau_{1}<\tau \leqslant \tau_{2}\end{cases} \\
k_{i j}(\tau)= \begin{cases}k_{1}(\tau), & 0<\tau \leqslant \tau_{1} \\
k_{2}(\tau), & \tau_{1}<\tau \leqslant \tau_{2}\end{cases}
\end{gathered}
$$

where $\tau=\varepsilon t$ is the "slow time"; $\varepsilon$ is a small constant parameter.

For the case considered here, the masses of each element are assummed to be constant and damping is neglected, the equations of the system are transferred into the principal coordinates or regular coordinates, so that the analytical method is the same as the linear one. The equation of the first principal coordinate can be expressed as follow:

$$
m_{1} \frac{d^{2} q_{1}}{d t^{2}}+k_{1} q_{1}=f_{1}\left(\tau, \theta, q_{1}, \frac{d q_{1}}{d t}\right)
$$

The first approximate solution of above equation according to the asymptotic method [6] can be written as follow:

$$
\begin{aligned}
q_{1}= & a \cos (s \varphi+\Psi)+\varepsilon u_{1}(\tau, \theta, s \varphi+\Psi) \\
& +\varepsilon^{2} u_{2}(\tau, \theta, s \varphi+\Psi)+\cdots
\end{aligned}
$$


where $u_{1}$ and $u_{2}$ are periodic functions of angles $\theta$ and $s \varphi+\Psi$ with the period of $2 \pi$, while $\varphi=\theta / r ; s$ and $r$ are reciprocal prime numbers.

The amplitude $a$ and phase $\Psi$ are the solutions of [6]

$$
\begin{aligned}
\frac{d a}{d t}= & \varepsilon A_{1}(\tau, a, \Psi)+\varepsilon^{2} A_{2}(\tau, a, \Psi)+\cdots \\
\frac{d \Psi}{d t}= & \omega(\tau)-\frac{s}{r} \nu(\tau)+\varepsilon B_{1}(\tau, a, \Psi) \\
& +\varepsilon^{2} B_{2}(\tau, a, \Psi)+\cdots
\end{aligned}
$$

where

$$
\begin{aligned}
A_{1}= & -\frac{a}{2 \omega m(\tau)} \frac{d[m(\tau) \omega]}{d \tau} \\
& +\sum_{\sigma} \frac{i \sigma(\omega r-s \nu) D_{1}-2 \omega D_{2}}{4 \omega^{2}-(\omega r-s \nu)^{2} \sigma^{2}} e^{i \sigma r \Psi} \\
B_{1}= & -\frac{1}{a} \sum_{\sigma} \omega \frac{i \sigma(\omega r-s \nu) D_{2}+2 \omega D_{1}}{4 \omega^{2}-(\omega r-s \nu)^{2} \sigma^{2}} e^{i \sigma r \Psi} \\
D_{1}= & \frac{1}{2 \pi^{2} m(\tau)} \cdot \int_{0}^{2 \pi} \int_{0}^{2 \pi} F_{0} e^{-i \sigma r \Psi} \\
& \cos (s \varphi+\Psi) d \theta d(s \varphi+\Psi) \\
D_{2}= & \frac{1}{2 \pi^{2} m(\tau)} \cdot \int_{0}^{2 \pi} \int_{0}^{2 \pi} F_{0} e^{-i \sigma r \Psi} \\
& \sin (s \varphi+\Psi) d \theta d(s \varphi+\Psi)
\end{aligned}
$$

and the $u_{1}$ can be gained as follows:

$$
\begin{gathered}
u_{1}(\tau, \theta, a, s \varphi+\Psi)=\sum_{\substack{m, n=-\infty \\
n+(m \pm 1) s \neq 0}}^{\infty} \\
\frac{F_{o m n}(\tau, a)}{m(\tau)\left[\omega^{2}-(m \omega+n \nu)^{2}\right]} e^{i[n \theta+m(s \varphi+\Psi)]}
\end{gathered}
$$

where

$$
\begin{aligned}
& F_{o m n}(\tau, a)=\frac{1}{4 \pi^{2}} \cdot \int_{0}^{2 \pi} \int_{0}^{2 \pi} \\
& F_{0}(\tau, a, \theta, s \varphi+\Psi) e^{-i[n \theta+m(s \varphi+\Psi)]} \\
& d \theta d(s \varphi+\Psi)
\end{aligned}
$$

where

$$
\sigma=\omega-\frac{s}{r} \nu, \nu=\frac{d \theta}{d t}, \omega=\sqrt{\frac{k(\tau)}{m(\tau)}} .
$$

Thus, the response of the system with slow-changing parameters can be obtained.

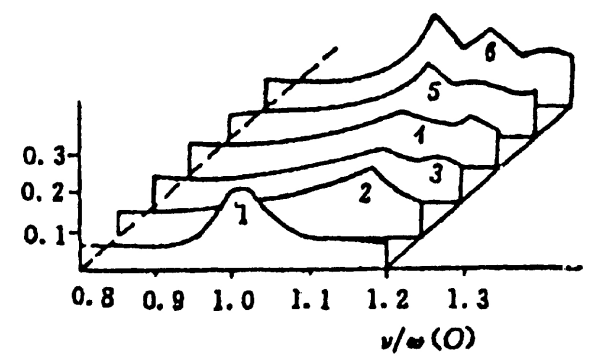

Fig. 2. The simulation curves of the system with different changing stiffness coefficients.

\section{Dynamic response and the experiment}

\subsection{The affection of the slow-changing parameters}

Suppose exciting frequency and stiffness change slowly as follow linear regularities

$$
\begin{aligned}
& \nu=\nu_{0}+\beta_{\nu} t \\
& k(\tau)=k_{0}+\delta_{k}(\tau)
\end{aligned}
$$

where $\beta_{\nu}$ is the slow rate of the exciting frequency.

The regularity of the amplitude, that varies slowly with respect to the exciting frequency, can be obtained by means of numerical calculation from Equation 7 .

Figure 1(a) shows the amplitude characteristic of a system with slow-changing exciting frequency while Fig. 1(b) shows the system with slow changing stiffness. In the process of the slow changing frequency, the resonant curve has no obvious soft or hard feature, but the unstable range is enlarged. From Fig. 1(b), it can be seen that the slow-changing stiffness not only affects the strength of the resonant curve but also affect the size of the stable region. The slow-changing parameters cause the unstable resonant curve to deviate. Therefore, the vibration characteristic of the system can be improved by means of controlling slow-changing parameters.

\subsection{Simulation of the system}

The motion equation of the system of inherent is (a system in this case)

$$
\begin{aligned}
{[M]\{\ddot{x}\}+[K(\tau)]\{x\}=} & \{E \sin \theta\} \\
& +\{\varepsilon f(\tau, \theta, x, \dot{x})\}
\end{aligned}
$$

The equation in the principal coordinates can be written as 

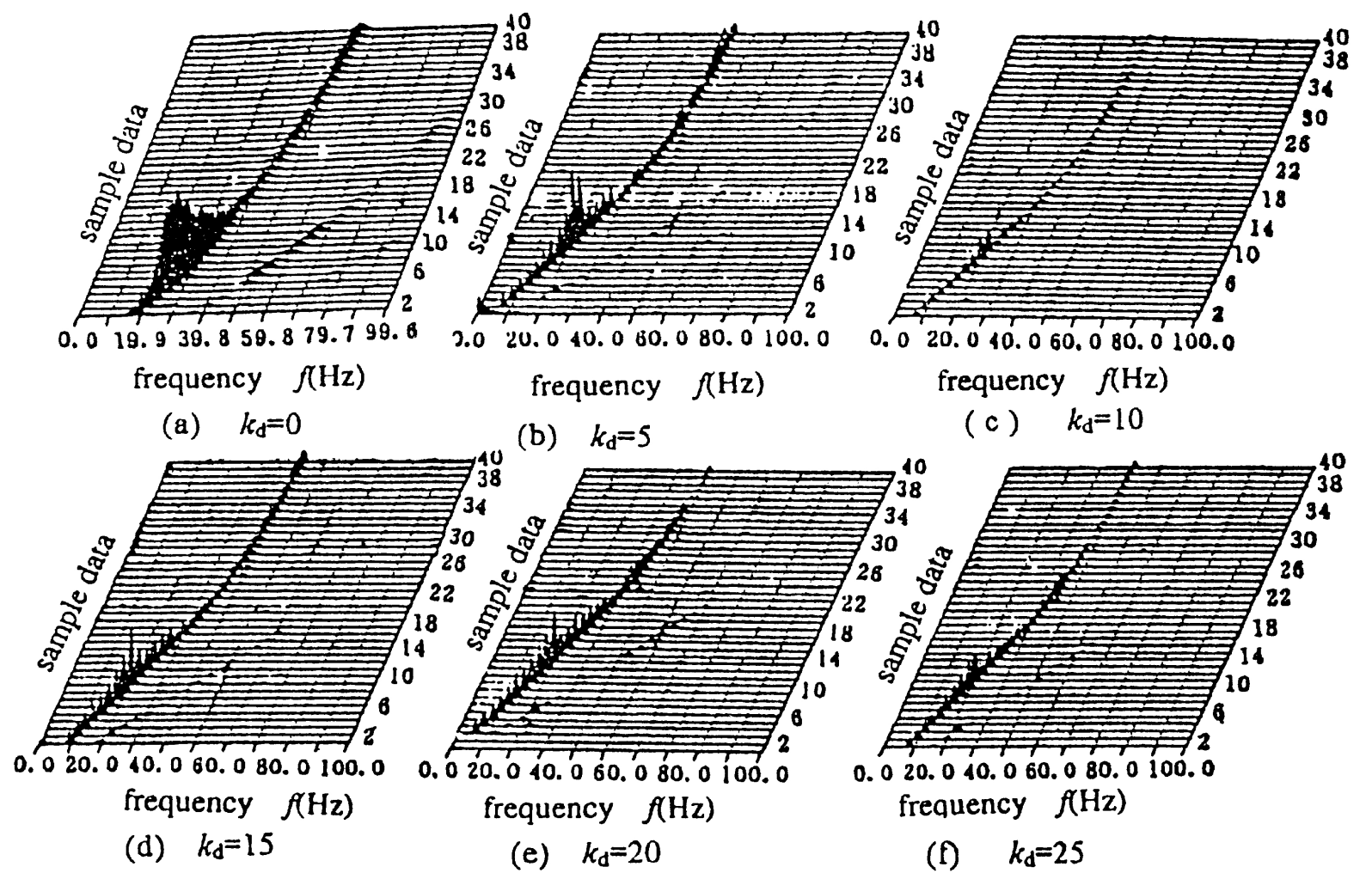

Fig. 3. The spectrums of the starting process with different changing stiffness coefficients.

$$
\frac{d^{2} q_{1}}{d t^{2}}+\omega_{1}^{2}(\tau) q_{1}=\varepsilon\left[E_{1} \sin \theta-c_{1} \frac{d q_{1}}{d t}\right]
$$

where

$$
\begin{aligned}
& \omega_{1}^{2}(\tau)=\frac{k_{1}(\tau)}{m_{1}} \quad \nu=\frac{d \theta}{d t} \\
& E=e \nu^{2}(\tau)=\varepsilon E_{1}
\end{aligned}
$$

Suppose the first approximate solution can be expressed as follows:

$$
q=a \cos \varphi
$$

$a$ and $\varphi$ can be obtained as follow equations according to the asymptotic method

$$
\begin{aligned}
& \frac{d a}{d t}=-\frac{\varepsilon E_{1}}{\omega+\nu} \cos \Psi-\frac{\varepsilon a}{2 \omega} \frac{d \omega}{d \tau}-\frac{\varepsilon c a}{2} \\
& \frac{d \varphi}{d t}=\omega-\nu+\frac{\varepsilon E_{1}}{a(\omega+\nu)} \sin \varphi
\end{aligned}
$$

In order to solve the Eq. (15) and (16) simply, in this paper, the changing of stiffness can be seen as a linear process with piecewise form:

$$
k(\tau)= \begin{cases}D_{1}+k_{d 1} \tau & 0<\tau \leqslant \tau_{1} \\ D_{2}+k_{d 2} \tau & 0<\tau_{1} \leqslant \tau_{2} \\ D_{3}+k_{d 3} \tau & 0<\tau_{2} \leqslant \tau_{3} \\ \cdots & \end{cases}
$$

where $k_{d}$ is the coefficient of the slow changing stiffness; $D_{1}, D_{2}$ and $D_{3}$ are constants.

When the values of the parameters are chosen as: $\varepsilon=$ $0.01, m=12.4 \mathrm{~kg} e=0.04 \mathrm{~m}, k(0)=3.2 \times 10^{6} \mathrm{~N} / \mathrm{m}$, $c_{1}=0.2 \mathrm{~s}^{-1}, \nu=628 \mathrm{~s}^{-1}$, the results can be obtained by using the fourth order Rung-Kutta method. In Fig. 2, curve $1,2,3,4,5,6$ are the simulation results when the $k_{d}$ takes the value of $0,5,10,15,20,25$, respectively. It is show that when $k_{d}=10$, the vibrating amplitude is the smallest. These cuves illustrate that we can be seen that we can restrain the vibration effectively in the starting and breaking process of the rotor system by choosing different slow changing stiffness coefficients.

\subsection{Experimental results}

Figure 3 is the experimental result of a rotor system with slow changing stiffness support in an intelligent structure. The experiment parameters are the same as 
the simulation ones. Figure 3(a) shows the spectrums of the starting process with constant stiffness, while Fig. 3(b) (f) show the spectrums of the starting process with different slow changing stiffness coefficients. As can be seen, when $k_{d}=10$, the control effect is the best of all. This because the resonant amplitude is the smallest in this case. It is also shown that the vibration of rotor system can be controlled effectively by means of choosing the suitable slow-changing coefficient. Such a scheme could be implemented by an intelligent structure system.

\section{Conclusions}

The amplitude and natural frequency of a system with slow-changing parameters vary slowly with respect to time. The slow-changing parameters not only affect the strength of the resonance, but also affect the size of the stability region of the system. Furthermore, the slow-changing stiffness can cause the deviation of the unstable resonance curve. In order to solve the problem simply and improve the accuracy of the solution, the slow-changing parameters can be regarded as a nonlinear process with piecewise linear coefficients.

In addition, when the damping become small, the amplitude characteristic of the system appears to fluctuate, nevertheless, when the damping becomes large the amplitude of the system decrease rapidly, and the system is inclined to the stable case. This simulation and numerical experiment shows that the suitable slow-changing stiffness coefficient can restrain the amplitude of the system when it passes through the resonant region.

\section{Acknowledgments}

This work partially supported by the National Natural Science Foundation of China.

\section{References}

[1] B. Wen et al., Vibration characteristics of the nonlinear system with slow- changing parameters, Journal of Nonlinear Dynamics 5(2) (1998), 181-188, in Chinese.

[2] D.L. Bosley and J. Kevorkian, Adiabatic invariance and transient resonance in very slowly varying oscillator hamiltonian systems, SIAM Journal of Applied Mathematics 52 (1992), 494-527.

[3] J. Kevorkian, Resonance in a weakly nonlinear systems with slowly varying parameters, SIAM Journal of Applied Mathematics 62 (1980), 23-67.

[4] J. Kevorkian, Perturbation techniques for oscillatory systems with slowly varying coefficients, SIAM Review 29 (1985), 391-461.

[5] S. Bravo Yusete, On duffing oscillation with slowly varying parameters, Int. J. Nonlinear Mechanics 26(5) (1991), 671677.

[6] S.M. Shahruz and C.A. Tan, Response of linear slowly varying systems under external excitations, Journal of Sound and Vibration 131(2) (1989), 239-247.

[7] Y.A. Mitropoliski, The Unstable Process of the Nonlinear Vibration System, Science Press, China, 1958, pp. 21-36. 

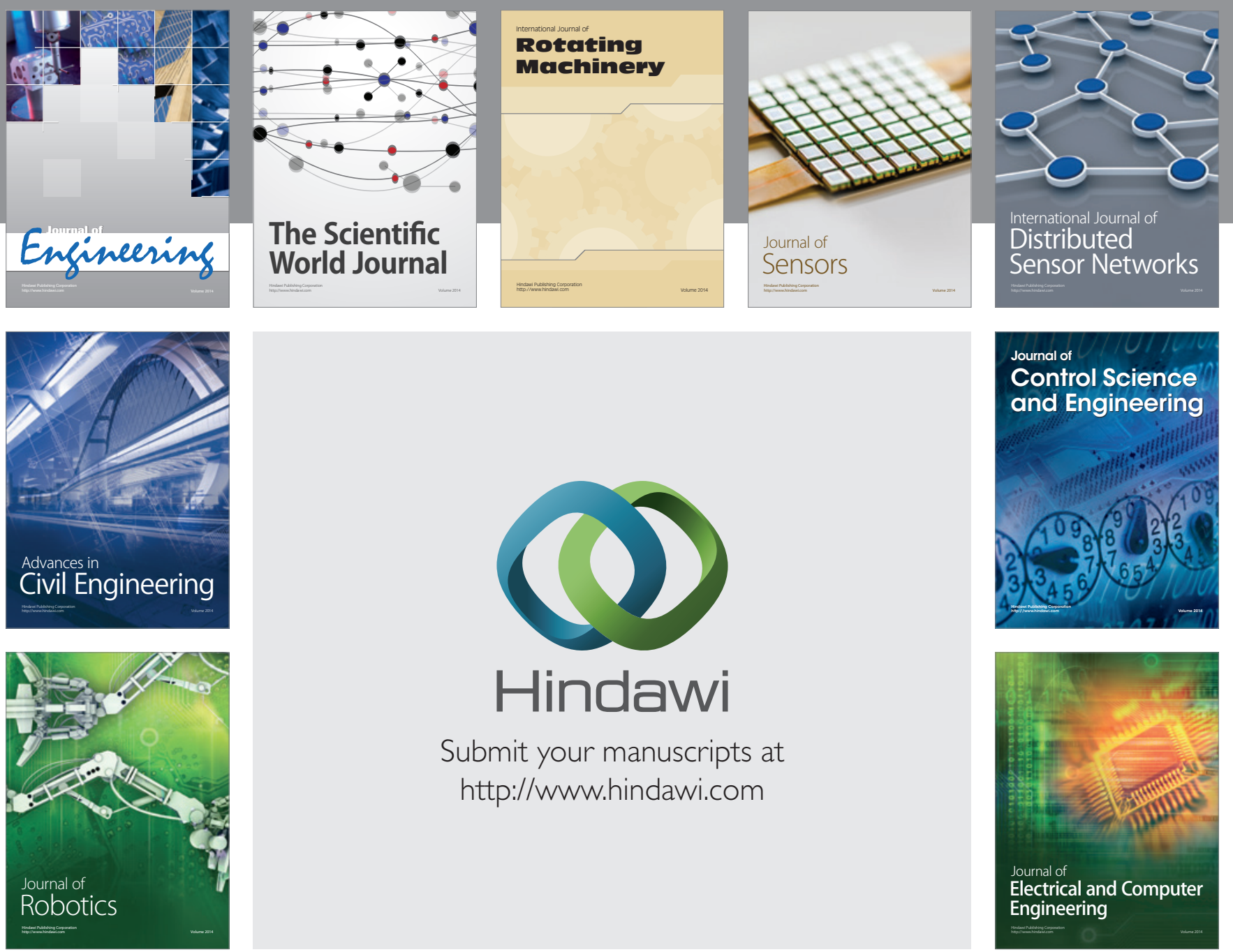

Submit your manuscripts at

http://www.hindawi.com
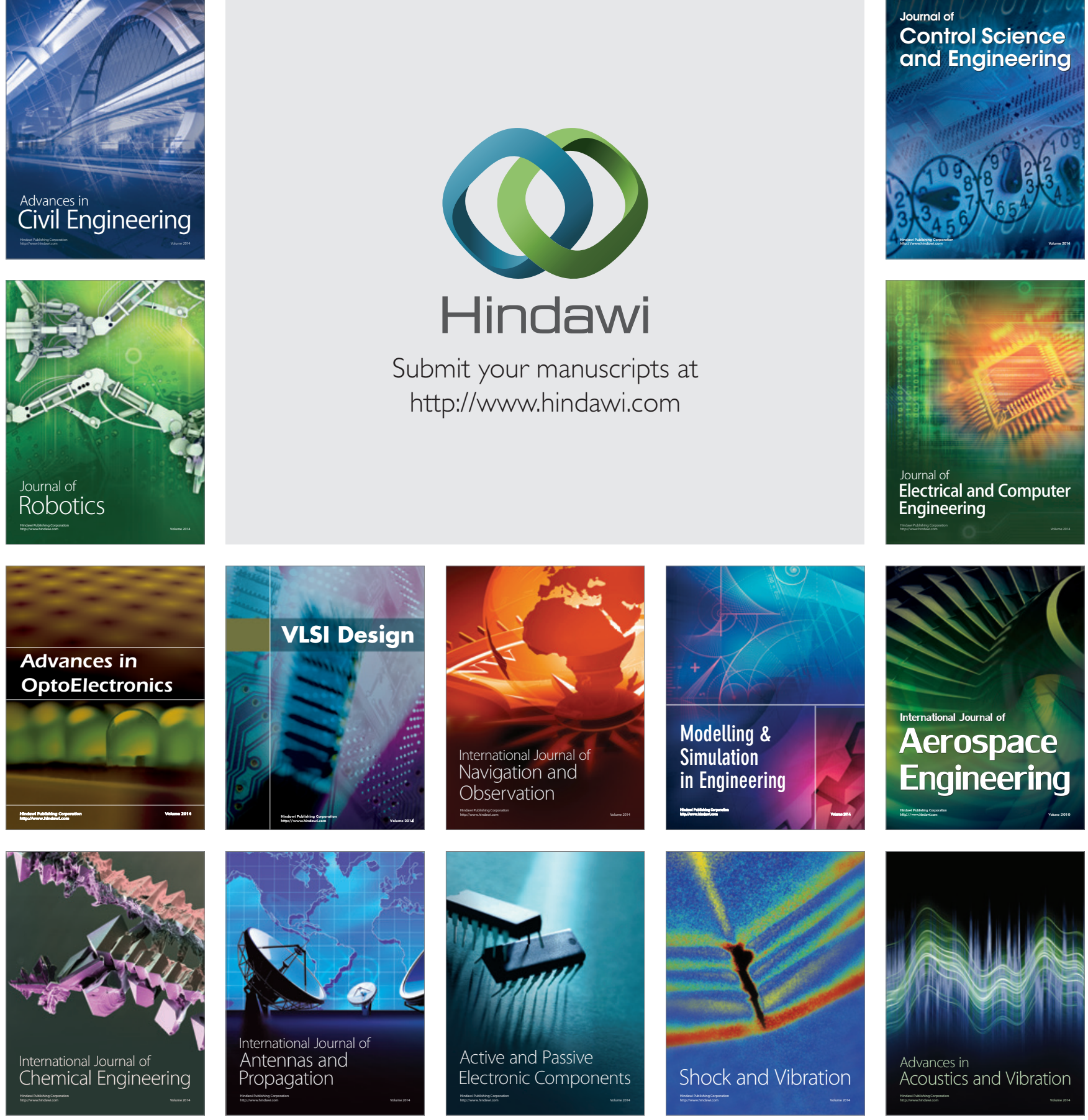